\title{
Young Investigator Awards
}

\section{Journal of}

Epilepsy and

Clinical

Neurophysiology

J Epilepsy Clin Neurophysiol 2006; 12(2):73-74

\section{Avaliação Eletrofisiológica in vitro de Drogas Antiepilépticas em Fatias Hipocampais Humanas Provenientes de Pacientes Portadores de Epilepsia do Lobo Temporal Refratária ao Tratamento Medicamentoso*}

André César da Silva, Elza Márcia Targas Yacubian, Américo Ceiki Sakamoto, Ricardo da Silva Centeno, Érika Sayuri Ueda, Leandro Leite Antônio, Leonardo Coutinho Faria, Margareth Rose Priel, Esper Abrão Cavalheiro

Laboratório de Neurologia Experimental, Universidade Federal de São Paulo/Escola Paulista de Medicina, SP, Brasil

Unitermos: drogas antiepilépticas, electrofisiologia in vitro, drogas resistentes.

\begin{abstract}
In vitro electrophysiological assessment of antiepileptic drugs in human hippocampal slices originating from patients with refractory temporal lobe epilepsy

The absence of a satisfactory response to antiepileptic drug (AED) therapy, is an unresolved problem in a significant number of epileptic patients. Mechanisms of intractability are not well understood but may include a combination of poor penetration of AED across a functionally altered blood-brain barrier owing to increased expression of multiple drug resistance transporters. Therefore, the aim of this work was to assess the in vitro efficacy of antiepileptic drugs through human hippocampal slices originating from patients with refractory temporal lobe epilepsy submitted to corticoamygdalohippocampectomy. Slices was prepared from a $1 \mathrm{~cm}^{3}$ block of the hippocampus body 30 min after resection. Briefly, hippocampal slices of $400 \mu \mathrm{M}$ thickness was cut coronally. Extracellular field potentials was recorded from the st. Granulosum of the dentate gyrus. The antiepileptic drugs added in the bath were Carbamazepine, Topiramate and Phenytoin. The phenytoin was effective reducing the hyperexcitability (polispikes) in $60 \%$ of the experiments $(n=5)$. On the other hand, the carbamazepine promoted a decrease in evoked epileptiform activity in $37,5 \%$ of the cases $(\mathrm{n}=8)$. The application of topiramate in the bath reduced in $30 \%$ the number of polispikes $(n=10)$. Our results showed that the phenytoin application resulted in a significant reduction in neuronal excitability, however, the carbamazepine and topiramate were not able to control of the hiperexcitability, suggesting that local neuronal alterations, as well changes in blood brain barrier, could be responsible for such behaviors.
\end{abstract}

Key words: antiepileptic drugs, in vitro electrophysiology, drug resistance.

\section{INTRODUÇÃO}

É classicamente descrito que uma parcela de pacientes portadores de Epilepsia do Lobo Temporal com Esclerose Mesial desenvolve um quadro de fármaco-resis- tência. Inúmeras hipóteses têm sido consideradas nesse aspecto. Algumas sugerem que essa fármaco-resistência poderia ser mediada por famílias de proteínas extrusoras presentes na membrana endotelial, o que resultaria em

\footnotetext{
* Trabalho premiado com o Prêmio "Jovem Pesquisador - Clínico e Cirúrgico", durante o XXXI Congresso Brasileiro de Epilepsia da LBE. Received Jun. 22, 2006; accepted Jun. 26, 2006.
} 
redução da concentração e portanto da eficácia da droga. Outras sugerem a ocorrência de alterações locais, como por exemplo, mudanças no sítio de ação, o que resultaria em perda da eficácia. Portanto, estudos para o entendimento dos mecanismos envolvidos na resistência ao tratamento farmacológico são de grande valia para o desenvolvimento de fármacos mais eficazes.

\section{OBJETIVO}

Avaliar a eficácia de drogas antiepilépticas em fatias hipocampais mantidas in vitro provenientes de pacientes portadores de Epilepsia do Lobo Temporal com esclerose mesial refratária ao tratamento medicamentoso submetidos à corticoamigdalohipocampectomia.

\section{MATERIAL E MÉTODOS}

Após a ressecção cirúrgica, a estrutura hipocampal foi imediatamente submersa em Líquido Cefalorraquiano Artificial (LCRa) resfriado a $4^{\circ} \mathrm{C}$. Fatias de $400 \mu \mathrm{M}$ foram cortadas no plano coronal, usando um vibrátomo, a partir de um bloco de $1 \mathrm{~cm}^{3}$ do corpo do hipocampo. Em seguida, as fatias foram colocadas numa câmara de estabilização constantemente oxigenada com mistura carbogênica $\left(95 \% \mathrm{O}_{2}, 5 \% \mathrm{CO}_{2}\right)$, em temperatura ambiente, por aproximadamente $2 \mathrm{~h}$. O LCRa tinha como composição (em mM) : $\mathrm{NaCl} 124 ; \mathrm{KCl} 4 ; \mathrm{Ca} \mathrm{Cl}_{2} 2 ; \mathrm{NaH}_{2} \mathrm{PO}_{4}$ 1.24; $\mathrm{MgSO}_{4}$ 1.3; $\mathrm{NaHCO}_{3} 26$ e glicose 10 . Na câmara de registro, a temperatura foi mantida a $35^{\circ} \mathrm{C}$. Os microeletrodos foram estirados utilizando capilares de $1.5 \mathrm{~mm}$ feitos de vidro borosilicato preenchidos com $\mathrm{NaCl} 1 \mathrm{M}$ (Resistência = 3-5 MW). Os potenciais de campo foram obtidos no st. Granulosum em diferentes áreas do giro dentado. Nos experimentos em que não houveram a ocorrência de atividade epileptiforme inicial, uma condição de hiperexcitabilidade foi induzida através da aplicação de um antagonista GABAérgico (bicuculina $10 \mu \mathrm{M}$ ) combinado ao aumento da concentração do potássio (7,5 mM) extracelular do LCRa. As drogas antiepilépticas adicionadas ao banho foram: Carbamazepina $50 \mu \mathrm{M}$, Topiramato $100 \mu \mathrm{M}$, Fenitoína $50 \mu \mathrm{M}$.

\section{RESULTADOS}

A Fenitoína foi efetiva reduzindo a hiperexcitabilidade do tecido (múltiplas populações de espículas) em $60 \%$ dos experimentos $(\mathrm{n}=15)$. Já a Carbamazepina promoveu a redução da atividade epileptiforme evocada em 37,5\% dos casos estudados $(n=8)$. Com a perfusão de Topiramato houve uma redução no número de espículas em apenas $30 \%$ das amostras estudadas $(\mathrm{n}=10)$.

\section{CONCLUSÕES}

Nosso estudo mostrou que, apesar da fármaco-resistência apresentada pelos pacientes, a aplicação in vitro de drogas antiepilépticas, como a fenitoína, resultou em uma redução significativa da excitabilidade do tecido. Por outro lado, a carbamazepina e o topiramato não foram eficazes no controle da hiperexcitabilidade, sugerindo que tanto alterações neuronais locais, como alterações na barreira hematoencefálica poderiam ser as responsáveis por tais comportamentos.

\author{
Endereço para correspondência: \\ André César da Silva \\ Rua Botucatu, 862 - Ed. José Leal Prado - Vila Clementino \\ CEP 04023-900, São Paulo, SP, Brasil \\ Fone: (11) 5576-4508 \\ E-mail: andre.nexp@epm.br
}

